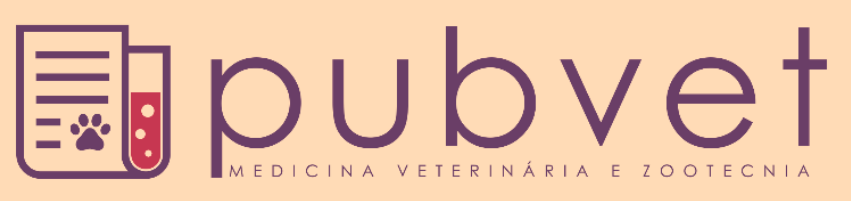

https://doi.org/10.31533/pubvet.v14n5a573.1-8

\title{
Alopecia X em cães: revisão
}

\author{
Adriana Leão de Carvalho Lima Gondim ${ }^{1} \bullet$ (D), Adjanna Karla Leite Araujo ${ }^{2} \bullet$ (D)
}

${ }^{I}$ Médica veterinária graduada pela Universidade Federal Rural de Pernambuco - UFRPE, Pós-graduada em Dermatologia Veterinária de Pequenos Animais pela instituição Qualittas - Universidade Castelo Branco. E-mail: adrianalclg@gmail.com ${ }^{2}$ Médica veterinária graduada pela Universidade Federal de Campina Grande - Campus de Patos/PB, Pós-graduada em Dermatologia Veterinária de Pequenos Animais pelas instituições Qualittas - Universidade Castelo Branco e Equalis, Especialização em Parasitologia Clínica e Saúde Pública pela UPE, Mestrado em Ciências Veterinárias pela Universidade Estadual do Ceará e pelo Centro de Pesquisa Aggeu Magalhães - FIOCRUZ-PE. E-mail: adjanna_leite@hotmail.com

Resumo. A alopecia em cães é um achado frequente nas consultas dermatológicas veterinárias, podendo ser causada por várias doenças cutâneas e até sistêmicas. A alopecia $\mathrm{X}$ é uma dermatopatia, que ocorre com mais frequência em raças nórdicas, especialmente em cães da raça Spitz Alemão, sobre a qual, atualmente, não há muito conhecimento científico. Sua patogenia ainda não foi bem esclarecida e atualmente acredita-se que seja causada por um componente hereditário associado a alterações de sensibilidade a receptores hormonais dos folículos pilosos. O tratamento mais utilizado é a castração desses animais, associando a administração de algumas drogas em determinados casos e o diagnóstico é feito observando-se a resposta do paciente ao tratamento instituído. Por ser uma doença sobre a qual não há muita literatura publicada, esse artigo tem como objetivo reunir informações importantes sobre esta dermatopatia.

Palavras chave: dermatologia, alopecia, alopecia X, ciclo piloso, cão

\section{Alopecia X in dogs: review}

Abstract. Alopecia in dogs is a frequent finding at veterinary dermatological consultations, may be caused by various skin diseases and even systemic. Alopecia $\mathrm{X}$ is a dermatopathy that occurs most often in Nordic breeds, especially in German Spitz dogs, about which there is not much scientific knowledge today. Its pathogenesis has not been well understood and is currently believed to be caused by an inherited component associated with changes in sensitivity to hair follicle hormone receptors. The most used treatment is the castration of these animals, associating the administration of some drugs in certain cases and the diagnosis is made by observing the patient's response to the treatment instituted. Being a disease about which there is not much published literature, this article aims to gather important information about this dermatopathy.

Keywords: dermatology, alopecia, alopecia X, hair cycle, dog

\section{Alopecia X en perros: revisión}

Resumen. La alopecia en los perros es un hallazgo frecuente en las consultas dermatológicas veterinarias, y puede ser causada por diversas enfermedades de la piel e incluso sistémicas. La alopecia $\mathrm{X}$ es una dermatopatía que ocurre con mayor frecuencia en las razas nórdicas, especialmente en los perros Spitz alemán, sobre el cual no hay mucho conocimiento científico en la actualidad. Su patogenia no ha sido bien entendida y actualmente se cree que es causada por un componente heredado asociado con cambios en la sensibilidad a los receptores de la hormona del folículo piloso. El tratamiento más utilizado es la castración de estos animales, asociando la administración de algunos 
medicamentos en ciertos casos y el diagnóstico se realiza observando la respuesta del paciente al tratamiento instituido. Al tratarse de una enfermedad sobre la cual no hay mucha literatura publicada, este artículo tiene como objetivo recopilar información importante sobre esta dermatopatía.

Palabras clave: dermatología, alopecia, alopecia X, ciclo piloso, perro

\section{Introdução}

Alopecia é a ausência de pelos em áreas que deveriam ser cobertas, devido a variadas causas, sendo comumente encontrada em diversas dermatopatias (Nuttal, 2010). O termo "alopecia X" ou aprisionamento do folículo piloso é utilizado para definir um conjunto de manifestações cutâneas em cães, como alopecia não inflamatória, bilateral, simétrica, normalmente sem prurido associado (Gross et al., 2008; Paradis, 2012). Além desses achados clínicos, o animal acometido apresenta pelagem sem brilho, que se desprende com facilidade da pele, melanodermia, além de outras alterações clínicas inespecíficas, quadro semelhante a diversas doenças endócrinas que também causam alopecia, porém sem sinais sistêmicos (Gross et al., 2008; Melián, 2004; Nuttal, 2010; Paradis, 2012).

Esta dermatopatia já foi chamada de pseudo-cushing, síndrome da hiperplasia adrenal congênita, alopecia responsiva ao hormônio de crescimento, dermatose responsiva à castração, alopecia responsiva à biópsia, entre outros menos conhecidos e é mais comumente observada em raças nórdicas, especialmente na raça Spitz Alemão (Miller et al., 2013). A grande quantidade de denominações para doença, deve-se ao fato da resposta variada a diversos tipos de tratamento instituídos nos animais portadores, em busca das possíveis causas dessa afecção (Frank, 2013).

A patogenia da doença não é bem esclarecida, mas acredita-se que inclua um componente hereditário associado a alterações de sensibilidade a receptores hormonais dos folículos pilosos (Frank, 2013; Nuttal, 2010). Segundo Medleau et al. (2003) uma das hipóteses apresentadas para explicar a causa e patogênese da doença é que a causa do distúrbio seria pela esteroidogênese adrenal anormal, sendo uma variante discreta do hiperadrenocorticismo dependente da hipófise, outras sugerem que a doença se deve à deficiência de hormônio do crescimento.

Ainda não há estudos aprofundados sobre os métodos de tratamento para esta dermatopatias, sendo a castração, a administração de melatonina e trilostano os mais comumente utilizados, com índices de repilação que podem atingir até 85\% (Feldman et al., 2014; Frank, 2013; Peterson, 2007).

\section{Aspectos epidemiológicos da doença}

A alopecia X normalmente é observada em animais adultos, jovens, em média, entre 1 e 2 anos, em sua maioria macho e não castrado (Cerundolo et al., 2008; Cerundolo et al., 2004; Nuttal, 2010); porém, pode ocorrer em ambos os sexos, independentemente do animal ser castrado ou não (Ferrer, 2005; Nuttal, 2010). Já Patel \& Forsythe (2011) afirmam que a doença é mais frequentemente observada em cães castrados. Algumas raças nórdicas de cães que apresentam um manto piloso denso e duplo como Malamute do Alasca, Chow Chow, Husky Siberiano, Spitz Alemão e Samoieda são mais comumente acometidas, porém a doença pode ser diagnosticada em cães de qualquer raça (Ferrer, 2005; Frank, 2013; Frank et al., 2004; Nuttal, 2010; Scott et al., 2001).

\section{O pelo e o ciclo piloso}

O crescimento do pelo é cíclico, compreendendo uma fase de crescimento ativo, uma fase transitória e uma fase de repouso (Patel \& Forsythe, 2010). O ciclo piloso (Figura 1) consiste em uma série de eventos de crescimento, regressão e remodelação ritmicamente recorrentes do pelo, controlados por uma complexa interação entre sinais clínicos do microambiente folicular e fatores sistêmicos importantes como fatores genéticos, hormonais e ambientais. Tal ciclo é composto pelas fases catágena, telógena, exógena e anágena. A fase catágena é caracterizada pela regressão do folículo piloso, a fase telógena consiste no período de repouso do folículo, a exógena é representada pela queda da haste pilosa e a fase anágena é a fase ativa do crescimento piloso (Brunner et al., 2017). 
Há grandes diferenças no ciclo piloso, principalmente nas fases telógena e anágena, nas diversas raças de cães (Baptista, 2017). Cães de raça, como Poodle e Bichon Frisé, apresentam ciclos predominantemente anágenos, e raças com revestimento de pelos do tipo "pelúcia", como Chow Chow, Malamute do Alasca e Spitz Alemão, apresentam o ciclo de pelos predominantemente telógeno (Patel $\&$ Forsythe, 2010). Normalmente, esse processo é muito bem equilibrado, de modo que o animal esteja coberto de pelos durante todo tempo (Hill, 2005). A substituição de pelos em cães ocorre em padrão de mosaico, ou seja, em uma mesma área cutânea há folículos pilosos nos três estágios do ciclo (Patel \& Forsythe, 2010).

Existem vários fatores que controlam tal crescimento, podendo ser resumidos como fatores intrínsecos e extrínsecos. Os primeiros são os fatores produzidos por células epiteliais e mesenquimais no folículo piloso e ao seu redor, como as citocinas, fatores de crescimento, hormônios, neuropeptídios e moléculas de adesão. Os fatores extrínsecos são aqueles produzidos por outros órgãos além da pele, como os hormônios da tireoide e os hormônios sexuais, além dos fatores externos, como a temperatura, fricção e terapia medicamentosa (Patel \& Forsythe, 2010).

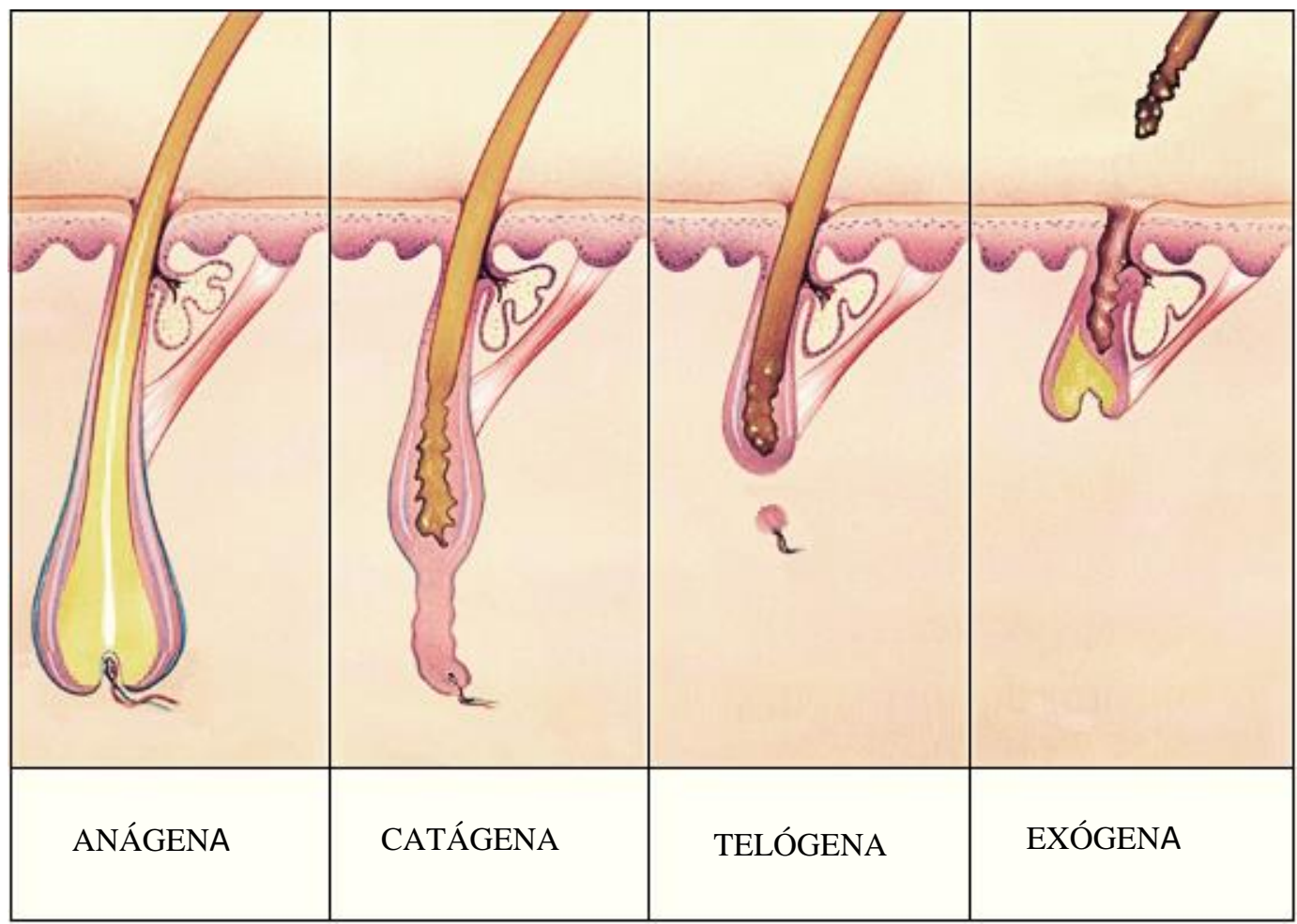

Figura 1: Fases do ciclo piloso. Fonte: Adaptado pelo autor.

$\mathrm{Na}$ alopecia $\mathrm{X}$, é relatado pelos tutores a falha na troca da pelagem infantil, o que sugere uma perturbação precoce do ciclo piloso e pode ser um importante elemento auxiliar no diagnóstico da doença (Baptista, 2017). Segundo Hill (2005), em animais portadores de doenças endócrinas como hiperadrenocorticismo e hipotireoidismo e em portadores de alopecia X, o ciclo piloso é interrompido na fase telógena, fazendo com que os pelos sofram queda sem serem substituídos.

\section{Patogenia}

Umas das primeiras teorias relacionava a doença à deficiência adulta do hormônio do crescimento, principalmente devido aos efeitos benéficos da aplicação deste hormônio em alguns cães afetados; porém, estudos realizados em cães com alopecia $X$, no qual esses animais eram submetidos a um teste de estimulação com xilazina e clonidina, agonistas alfa-adrenérgicos que estimulam a liberação de hormônio de crescimento pelo hipotálamo, apresentaram resultados pouco consistentes. Além disso, ao realizar a suplementação desses animais com hormônio do crescimento não houve melhora do quadro, concluindo assim que a deficiência adulta deste hormônio seria uma causa altamente improvável da doença (Feldman \& Nelson, 2004; Frank, 2013). A partir da década de 90, foram iniciados diversos 
estudos investigativos sobre hormônios esteroidais adrenocorticais na raça Spitz Alemão e em nenhum deles observou-se relação direta com a doença (Cerundolo et al., 2007; Frank, 2013; Frank et al., 2004; Frank et al., 2003; Shibata et al., 2005).

A causa e a patogênese da alopecia $X$, ainda hoje é desconhecida. Acredita-se que haja uma predisposição genética que resulta em falha na produção hormonal ou em uma ação hormonal deficiente sobre o folículo piloso (Feldman \& Nelson, 2004; Ferrer, 2005; Cerundolo et al., 2008). Algumas manifestações corroboram a hipótese de que a ação deficiente da produção de hormônios sexuais pode ser responsável pela alopecia X, tais como o crescimento do pelo em cães após a castração ou tratamento com princípios ativos que afetam a produção de hormônios sexuais e, que diminuem os níveis de hormônios (Cerundolo et al. 2008; Frank et al., 2006). A maior predisposição que as raças nórdicas apresentam para a doença, a manifestação clínica relativamente precoce e a análise genealógica dos cães afetados sugerem que a doença tenha caráter hereditário, porém o modo de transmissão hereditária ainda não foi elucidado, já que se observou poucas alterações significativas nos genes dos animais portadores (Gross et al., 2005; Mausberg et al., 2007).

Em sequenciamento genético realizado a partir de amostras de pele de cães da raça Spitz Alemão com alopecia $\mathrm{X}$ foram identificadas alterações em dez genes envolvidos na síntese de hormônios sexuais, vitamina $\mathrm{D}$ e metabolismo da melatonina, reforçando a hipótese de que o metabolismo desses hormônios esteja alterado localmente na pele de indivíduos portadores da doença (Brunner et al., 2017). Além disso, constatou-se uma hiper-regulação de enzimas envolvidas na degradação plasmática e dérmica da melatonina, o que explicaria a resposta terapêutica do paciente à administração de melatonina, compensando parcialmente esse aumento da degradação local (Frank, 2006; Brunner et al., 2017).

\section{Sinais clínicos}

A doença caracteriza-se pela interrupção do ciclo piloso nos animais acometidos. Normalmente observa-se alopecia progressiva, marcadamente bilateral e simétrica, não inflamatória e com hiperpigmentação da pele (Frank et al., 2006; Nuttal et al., 2010). O desenvolvimento da afecção é lento inicialmente e, o animal apresenta apenas a perda dos pelos primários, retendo os secundários, principalmente nas áreas de maior atrito como em volta do pescoço, cauda, região dorso-caudal, períneo e área caudal das coxas. Após um tempo, a alopecia progride e o pelo dessas regiões cai completamente, surgindo amplas áreas alopécicas. Em alguns pacientes, também se observa queda dos pelos primários do tronco e da cabeça e, extremidades distais dos membros são preservadas, sendo essa uma condição quase que patognomônica da doença (Frank et al., 2004; Ferrer, 2005; Gross et al., 2005; Müntener et al., 2012),

Uma característica clínica interessante é o recrescimento piloso em áreas de trauma ou biópsia cutânea (Gross et al., 2005; Patel \& Forsythe, 2010; Muntener et al., 2012). Na maioria dos casos o animal não apresenta prurido, a menos que ocorra infecção bacteriana secundária ou alteração da queratinização, embora esses achados sejam raros (Cerundolo et al., 2008). Outro aspecto importante a ser observado é que esses animais são absolutamente saudáveis, sem qualquer alteração sistêmica, porém como o padrão de lesão da doença é similar ao apresentado em animais portadores de doenças endócrinas, deve-se sempre investigar e excluir as endocrinopatias que causam alterações dermatológicas como hipotireoidismo, hiperadrenocorticismo e distúrbios gonadais, sendo de exclusão, o diagnóstico da alopecia X (Cerundolo et al. 2007; Frank, 2013).

\section{Diagnóstico e diagnóstico diferencial}

$\mathrm{O}$ diagnóstico da alopecia $\mathrm{X}$ é primordialmente clínico e baseia-se em eliminar a possibilidade de outras doenças endócrinas que também causem alopecia, neoplasias gonadais funcionais, adenite sebácea, eflúvio telogênico e outras displasias foliculares (Ferrer, 2005; Nelson \& Couto, 2015). Deve ser levado em consideração fatores como raça, idade, localização das áreas alopécicas e ausência de prurido e de manifestações clínicas sistêmicas (Cerundolo et al., 2004). Cerundolo et al. (2004), estabelece uma lista de critérios para o diagnóstico de alopecia $X$, que são eles: predisposição racial, idade de início das lesões entre 2 e 6 anos, o paciente apresenta perda progressiva da pelagem truncal e/ou textura lanosa do pelo com ou sem hiperpigmentação cutânea, ausência de sinais clínicos sistêmicos 
e alterações em exames laboratoriais como hemograma e dosagens bioquímicas, função tireoidiana normal, aumento na razão cortisol/creatinina urinária na maioria das amostras de urina coletada por um período de 10 dias, leve ou moderada supressão da razão cortisol/creatinina urinária após teste de supressão com baixa dose de dexametasona e achados histopatológicos de queratinização tricolemal dos folículos pilosos.

Segundo Gross et al. (2005) os achados das biópsias de pele de cães com alopecias não inflamatórias são frequentemente inespecíficos e incapazes de diferenciar a alopecia $\mathrm{X}$ das demais doenças que causam esse tipo de alteração na pele. Em todas essas doenças, observa-se uma discreta atrofia e hiperqueratose ortoqueratótica da epiderme, atrofia folicular, hiperpigmentação epidérmica, predomínio das fases telógena e catágena dos folículos pilosos, dilatação do infundíbulo folicular, com poucas ou nenhuma haste de pelo, atrofia epidérmica e atrofia das glândulas sebáceas (Patel \& Forsythe, 2010). Todavia, a presença de "folículos em chama" que são caracterizados por queratinização tricolemal excessiva é observada com mais frequência em cães com alopecia $\mathrm{X}$ do que em outras dermatopatias (Gross et al., 2005; Muntener et al., 2012).

A resposta à terapia consiste em outra forma de diagnóstico, embora alguns animais apresentem melhora com determinado princípio ativo e outros com outro e, ainda existem aqueles que não respondem a qualquer terapia médica ou cirúrgica, como a orquiectomia (Cerundolo et al., 2008). As doenças de origem hormonal que causam alopecia não inflamatória, como o hiperadrenocorticismo, o hipotireoidismo e o hiperestrogenismo são consideradas os principais diagnósticos diferenciais da alopecia X; porém, essas endocrinopatias causam diversas alterações sistêmicas e normalmente acometem animais mais velhos. De qualquer forma, testes hormonais específicos para descartar cada uma dessas doenças devem ser realizados (Paradis \& Cerundolo, 2003; Brunner et al., 2017).

\section{Tratamento}

Os tratamentos mais utilizados atualmente são a castração, a administração de melatonina e administração do trilostano. Os pacientes tratados apresentam repilação com índices que variam entre 40 e $85 \%$. Outra forma de tratamento que vem sendo utilizado é o microagulhamento com índices de repilação que pode chegar até $90 \%$.

Segundo Patel \& Forsythe (2010) e Nuttal et al. (2010), a castração resulta em crescimento de novos pelos tanto em machos quanto em fêmeas e, deve ser recomendada como o tratamento de primeira escolha. Este procedimento leva ao crescimento total ou parcial dos pelos em cerca de 4 a 8 semanas. Embora, cerca de 15\% dos casos apresentam recidivas anos depois. Segundo Cerundolo et al. (2008) e Scott et al. (2002), a castração tem sido o tratamento de escolha em cães machos, com parcial ou total repilação. Em animais castrados, por razões de custo e segurança, atualmente a melatonina é considerada o tratamento de escolha pela maioria dos dermatologistas veterinários (Patel \& Forsythe, 2010). Nos mamíferos, a melatonina está relacionada com a função tireoidiana, sistema reprodutivo, regulação de fotoperíodo, indução do sono, manutenção da pelagem, termorregulação e com a adaptação do animal às mudanças ambientais sazonais (Lincoln, 2006; Rajaratnam et al., 2003).

Em cães, sua função fisiológica ainda não está totalmente compreendida; porém, sabe-se que nesses animais o ritmo de produção da melatonina é noturno e que esse hormônio atua promovendo a fase anágena no folículo piloso. Sendo assim, a administração de melatonina deve ser realizada preferencialmente no período noturno (Frank et al., 2004); porém, de acordo com Medleau et al. (2003), a melatonina deve ser usada para o tratamento da alopecia X na dose de 3 a $12 \mathrm{mg}$ totais para cada cão em intervalos de 8 a 24 horas até que se obtenha o máximo crescimento de novos pelos, apresentando, esse protocolo, $50 \%$ de eficácia. Passado esse período, deve-se administrar a dose de 3 a $6 \mathrm{mg}$, por via oral, em intervalos de 24 horas, durante dois meses. Em seguida, administra-se a melatonina duas vezes por semana como terapia de manutenção. Já segundo Patel \& Forsythe (2010) a dose normalmente utilizada é de $3 \mathrm{mg} / \mathrm{kg}$, duas ou três vezes a dia, durante três meses, podendo observar crescimento de novos pelos em cerca de um terço dos casos com este tratamento.

Os tratamentos com trilostano utilizados em casos de alopecia $\mathrm{X}$ publicados até o momento possuem respostas terapêuticas imprevisíveis, mesmo assim observa-se um recrescimento piloso importante após seu uso em diferentes protocolos terapêuticos (Cerundolo et al., 2004; Frank, 2013). Essa repilação pode 
ser explicada, provavelmente, pela ação do trilostano a nível de folículo piloso, já que este possui a capacidade de sintetizar hormônios esteroidais (Ohnemus et al., 2006). Segundo Cerundolo et al. (2008), Medleau et al. (2003) e Nuttal et al. (2010) a dose recomendada varia de $3 \mathrm{a} 5 \mathrm{mg} / \mathrm{kg}$, a cada 24 horas, por via oral, durante vários meses, podendo-se diminuir a frequência de administração para 2 a 3 vezes por semana, após o crescimento do pelo, de acordo com cada caso. Embora seja bem tolerado, há relatos de que o trilostano pode causar morte súbita em cães com problemas cardíacos concomitantes e também é contraindicado em fêmeas caninas gestantes e lactantes, cães com doenças hepáticas primárias e com insuficiência renal (Medleau et al., 2003).

O microagulhamento é uma técnica considerada inovadora e disponível no Brasil, mostrou-se eficiente no tratamento da alopecia X. Tal técnica constitui-se em um estímulo físico realizado através de uma caneta dérmica diretamente na pele dos animais (Baptista, 2017; Stoll et al., 2015). Estudos realizados nos últimos anos evidenciaram que os micros traumas causados por essa técnica foram capazes de estimular o folículo piloso (Jeong et al., 2012; Kim et al., 2012). O tratamento possivelmente age ativando células-tronco das raízes dos folículos pilosos, induzindo novo ciclo de crescimento piloso (Stoll et al., 2015). Outra medicação que vem sendo utilizada para o tratamento da doença, apesar da sua ação no folículo piloso não estar ainda totalmente compreendido, é o acetato de deslorelina (Suprelorin 4,7 mg ${ }^{\circledR}$ ) via implante inter escapular subcutâneo. Estudos realizados em cães não castrados diagnosticados com alopecia $\mathrm{X}$ submetidos ao tratamento com tal medicamento apresentaram um bom recrescimento piloso, em um período variando de dois a quatro meses após sua aplicação (Albanese et al., 2014). Além disso, recomenda-se o uso dos mesmos produtos comerciais voltados para o tratamento da dermatite atópica, com o objetivo de promover a hidratação da pele e pelame que restou do animal, já que os animais acometidos pela doença apresentam intenso ressecamento cutâneo (Cerundolo et al., 2004; Olivry et al., 2010). Como a alopecia X, apesar de ser uma dermatopatia, é uma condição puramente estética, não comprometendo a saúde do animal e os efeitos secundários à terapia escolhida podem ser superiores aos benefícios, não fazer qualquer tipo de tratamento também é uma opção aceitável (Medleau et al., 2003; Nuttal et al., 2010).

\section{Conclusão}

A alopecia em cães é um sinal clínico observado com muita frequência na rotina de atendimento clínico dos dermatologistas veterinários, podendo possuir inúmeras causas. A alopecia X é uma dermatopatia que acomete os cães sendo mais comumente observada em raças nórdicas, não inflamatória, não pruriginosa e de etiopatogenia desconhecida sobre a qual ainda não existe estudos suficientes realizados.

Os animais acometidos normalmente não apresentam alterações sistêmicas, devendo-se portanto, avaliar os benefícios e malefícios do tratamento a ser instituído e o principal método de diagnóstico atualmente ainda é a resposta do paciente à castração, considerado o tratamento de primeira escolha nesses casos, ou à outra terapia instituída com o objetivo de estimular a repilação desses animais.

O médico veterinário deve estar ciente da ocorrência dessa doença, seus sinais clínicos e tratamentos descritos na literatura atualmente, para que oriente os tutores desses animais de forma correta.

\section{Referências}

Albanese, F., Malerba, E., Abramo, F., Miragliotta, V. \& Fracassi, F. (2014). Deslorelin for the treatment of hair cycle arrest in intact male dogs. Veterinary Dermatology, 25(6), 519-e88.

Baptista, A. B. M. V. (2017). Sinalização e desenvolvimento da Alopecia X em 171 cães da raça Spitz Alemão. Anais Do III Congresso Internacional Da Associação Brasileira de Endocrinologia Veterinária.

Brunner, M. A. T., Jagannathan, V., Waluk, D. P., Roosje, P., Linek, M., Panakova, L., Leeb, T., Wiener, D. J. \& Welle, M. M. (2017). Novel insights into the pathways regulating the canine hair cycle and their deregulation in alopecia X. PloS One, 12(10). https://doi.org/10.1371/journal.pone.0186469.

Cerundolo, R., Bensignor, E. \& Guaguére, E. (2008). Sex hormone dermatoses. In E. Guaguére, P. Prélaud, \& M. E. Craig (Eds.), A pratical guide in canine dermatology. Merial.

Cerundolo, R, Lloyd, D. H., Vaessen, M., Mol, J. A., Kooistra, H. S. \& Rijnberk, A. (2007). Alopecia 
in pomeranians and miniature poodles in association with high urinary corticoid: creatinine ratios and resistance to glucocorticoid feedback. Veterinary Record, 160(12), 393-397.

Cerundolo, R., Lloyd, D. H., Persechino, A., Evans, H. \& Cauvin, A. (2004). Treatment of canine Alopecia X with trilostane. Veterinary Dermatology, 15(5), 285-293.

Feldman, E. C., Nelson, R. W., Reusch, C. \& Scott-Moncrieff, J. C. (2014). Canine and feline endocrinology. Elsevier Health Sciences.

Frank, L.A. (2013). Endocrine and metabolic diseases. In W.H. Miller, C. E. Griffin, \& K. L. Campbel (Eds.), Muller \& Kirk's small animal dermatology (7th ed.). Elselvier Saunders.

Frank, L.A., Hnilica, K. A. \& Oliver, J. W. (2004). Adrenal steroid hormone concentrations in dogs with hair cycle arrest (Alopecia X) before and during treatment with melatonin and mitotane. Veterinary Dermatology, 15(5), 278-284. https://doi.org/https://doi.org/10.1111/j.1365-3164. 2004.00372.x.

Frank, Linda A, Donnell, R. L. \& Kania, S. A. (2006). Oestrogen receptor evaluation in Pomeranian dogs with hair cycle arrest (alopecia X) on melatonin supplementation. Veterinary Dermatology, 17(4), 252-258.

Frank, Linda A, Hnilica, K. A., Rohrbach, B. W. \& Oliver, J. W. (2003). Retrospective evaluation of sex hormones and steroid hormone intermediates in dogs with alopecia. Veterinary Dermatology, 14(2), 91-97.

Gross, T. L., Ihrke, P. J., Walder, E. J. \& Affolter, V. K. (2008). Skin diseases of the dog and cat: clinical and histopathologic diagnosis. John Wiley \& Sons.

Hill, P. (2005). Clinical Approach to Alopecia in Dogs-Will the Hair Grow Back. The Royal School Of Veterinary Studies, 263-268.

Jeong, K., Lee, Y. J., Kim, J. E., Park, Y. M., Kim, B. J. \& Kang, H. (2012). Repeated microneedle stimulation induce the enhanced expression of hair-growth-related genes. International Journal of Trichology, 4(2), 117-130.

Kim, B. J., Lim, Y. Y., Kim, H. M., Lee, Y. W., Won, C. H., Huh, C. H. \& Kang, H. (2012). Hair follicle regeneration in mice after wounding by microneedle roller. International Journal of Trichology, 4(2), $117-130$.

Lincoln, G. A. (2006). Decoding the nightly melatonin signal through circadian clockwork. Molecular and Cellular Endocrinology, 252(1-2), 69-73.

Mausberg, E.-M., Drögemüller, C., Dolf, G., Leeb, T., Rüfenacht, S. \& Welle, M. (2007). Evaluation of the CTSL2 gene as a candidate gene for alopecia $\mathrm{X}$ in Pomeranians and Keeshonden. Animal Biotechnology, 18(4), 291-296.

Medleau, L., Hnilica, K. A. \& Fagliari, G. S. (2003). Dermatologia de pequenos animais: atlas colorido e guia terapêutico. Roca.

Melián, C. (2004). Alopecia X in dogs. Proceedings of North American Veterinary Conference, 18, 371.

Miller, William Howard, Griffin, C. E., Campbell, K. L. \& Muller, G. H. (2013). Muller and Kirk's Small Animal Dermatology. Elsevier Health Sciences.

Müntener, T., Schuepbach-Regula, G., Frank, L., Rüfenacht, S. \& Welle, M. M. (2012). Canine noninflammatory alopecia: a comprehensive evaluation of common and distinguishing histological characteristics. Veterinary Dermatology, 23(3), 206-e44.

Nelson, R. W. \& Couto, C. G. (2015). Medicina interna de pequenos animais (Issue 1). Elsevier Editora.

Nuttal, T. (2010). Enfermedades cutaneas del perro y el gato. Grupoasis, Zaragoza, Espanha.

Ohnemus, U., Uenalan, M., Inzunza, J., Gustafsson, J.-A. \& Paus, R. (2006). The hair follicle as an estrogen target and source. Endocrine Reviews, 27(6), 677-706.

Olivry, T., DeBoer, D. J., Favrot, C., Jackson, H. A., Mueller, R. S., Nuttall, T. \& Prélaud, P. (2010). Treatment of canine atopic dermatitis: 2010 clinical practice guidelines from the International Task Force on Canine Atopic Dermatitis. Veterinary Dermatology, 21(3), 233-248.

Paradis, M. (2012). An approach to symmetrical alopecia in the dog. In BSAVA Manual of Canine and Feline Dermatology (pp. 91-102). BSAVA Library.

Patel, A. \& Forsythe, P. J. (2011). Dermatologia em pequenos animais. Elsevier Brasil. 
Peterson, M. E. (2007). Diagnosis of hyperadrenocorticism in dogs. Clinical Techniques in Small Animal Practice, 22(1), 2-11.

Rajaratnam, S. M. W., Dijk, D.-J., Middleton, B., Stone, B. M. \& Arendt, J. (2003). Melatonin phaseshifts human circadian rhythms with no evidence of changes in the duration of endogenous melatonin secretion or the 24-hour production of reproductive hormones. The Journal of Clinical Endocrinology \& Metabolism, 88(9), 4303-4309.

Scott, D. W., H., M. W. \& E., G. C. (2001). Mullerand Kirk's small animal dermatology. WB. Saunders.

Shibata, K., Koie, H. \& Nagata, M. (2005). Clinicopathologic and morphologic analysis of the adrenal gland in Pomeranians with non-illness alopecia. The Japanese Journal of Veterinary Dermatology, 11(3), 115-120.

Stoll, S., Dietlin, C. \& Nett-Mettler, C. S. (2015). Microneedling as a successful treatment for alopecia $\mathrm{X}$ in two $\mathrm{P}$ omeranian siblings. Veterinary Dermatology, 26(5), 387-388. https://doi.org/https://doi.org/10.1111/vde.12236.

Recebido: 28 de janeiro, 2020.

Aprovado: 20 de março, 2020.

Disponível online: 17 de junho, 2020.

Licenciamento: Este artigo é publicado na modalidade Acesso Aberto sob a licença Creative Commons Atribuição 4.0 (CC-BY 4.0), a qual permite uso irrestrito, distribuição, reprodução em qualquer meio, desde que o autor e a fonte sejam devidamente creditados. 